\title{
Für eine Hausarztmedizin mit Herz und Zähnen
}

\author{
Aus einer Vereinigung von 1000 Ärztinnen und Ärzten ohne Spezialarzttitel, die \\ sich für gleiche Rechte für Nichttitelträger einsetzte, wurde ein kämpferischer \\ Verein für den Erhalt des «Ambulatorium Schweiz».
}

Fooderatio Modicarum Procticarum
Foederatio Medicorum Practicorum

Dr. med. H.U. Bürke

Präsident FMP

Korrespondenz:

hansulrich.buerke@hin.ch

www.fmp-net.ch
Mit dem Reformprojekt GRAT, dem nachmaligen TARMED, drohte den praktizierenden Ärztinnen und Ärzten ohne Facharzttitel Mitte der 90er-Jahre eine massive Diskriminierung. Vorerst im Kanton Zürich schlossen sie sich deshalb zusammen und gründeten 1995 auf nationaler Ebene die Foederatio medico(a)rum practico(a)rum.

\section{Diskriminiert von den eigenen Kollegen}

Die titellosen Praktiker hatten es anfangs nicht leicht. Sie waren beispielsweise im Ärzteparlament unerwünscht. Immerhin erteilte die FMH der FMP den Auftrag, das Curriculum des praktizierenden Arztes zu erarbeiten. Nur dank der unermüdlichen, zeitweise heftig angefeindeten Arbeit von Kollegin Ingrid WylerBrem konnten die «Zweitklass-Mediziner» schliesslich nachweisen, dass sie sehr wohl eine gleichwertige, lediglich formal nicht genügende Weiterbildung hinter sich hatten. Übergangsbestimmungen im neuen FMPG (Freizügigkeitsgesetze für Medizinalberufe) schufen am Ende die Voraussetzungen zum nachträglichen Titelerwerb für alle, auch für die nicht in der FMP vereinten Kolleginnen und Kollegen.

\section{Zugehörigkeit und Eigenständigkeit}

2002 war mit der Umsetzung des FMPG die Titelfrage gelöst, Ende 2007 die Aufgabe der FMP abgeschlossen. Inzwischen ist die Mehrheit unserer Mitglieder längst auch bei der SGAM eingeschrieben, die uns auf unserem Weg unterstützt hatte. Eines aber haben wir in all den Jahren gelernt: Es ist wichtig, auf Entwicklungen und Gefahren aufmerksam zu machen, die auf der politischen Agenda grosser Fachgesellschaften fehlen. Die FMP setzt sich deshalb weiterhin für den Erhalt des «Ambulatorium Schweiz» ein. Sie ist eigenständig geblieben und hat, unterstützt durch Rechtsanwalt D. Staffelbach, einen ausgewiesenen Kenner des Gesundheitswesens, wiederholt pointiert Missstände in der Gesundheitspolitik offen gelegt.

\section{Missstände}

Der TARMED mit seinem Dignitätskonzept ist ein Konstrukt, das die FMP seit je her in Frage stellte. Diese Haltung fand bei den Grundversorgergesellschaften leider lange kein Echo, obwohl absehbar war, dass der Hausarzt entgegen aller Versprechungen im TARMED nicht die ihm zugedachte Bedeutung erlangen würde. Unsere ablehnende Haltung führte zur Zeit der TARMEDUrabstimmung bei der SGAM zu erheblicher Verstimmung. Nachträglich wird mancher eingestehen, dass die FMP mit ihrer kritischen Haltung Recht hatte. Heute ist die FMP in den entscheidenden Fragen solidarisch mit allen Grundversorgern. Davon zeugen unsere Auftritte an den 1.-April-Demonstrationen. Wir tragen den Dachverband «Hausärzte Schweiz» aktiv mit und sind in der Gründungskommission mit dem Präsidenten vertreten.

\section{Kritische und wachsame Partner bleiben}

Dennoch, die FMP wird weiterhin alle Projekte von FMH oder BAG, die die Arbeit des Hausarztes belasten, vehement bekämpfen. Davon zeugt unser Widerstand gegen das unselige Dignitätskonzept ebenso wie gegen das vom Ärzteparlament verabschiedete, der Basis aber weitgehend unbekannt gebliebene Projekt DINAMO mit seinen inakzeptablen administrativen Folgen: Erfassung und Auswertung des Patienten-Behandlungspfades durch den Hausarzt. Bedrohlich für den selbständigen Arzt ist auch der politische Druck, Netzwerke einzuführen. Dies wird die selbständigen Hausärzte massiv betreffen, weil Netzwerke nur mit grossem Kapital aufgebaut werden können. Ärzte und Ärztinnen werden so zu Angestellten. Der freie Beruf Arzt verschwindet.

Die Hausärzte sind aufgerufen, sich gegen alle Demontageversuche zur Wehr zu setzen und konstruktiv im Gesundheitswesen präsent zu bleiben. Die FMP steht allen Kolleginnen und Kollegen als eigenständige, selbst den eigenen Reihen gegenüber kritische Plattform offen. Sie bleibt die Hüterin des «Ambulatorium Schweiz». 